\title{
Overdesign e sviluppo del nuovo prodotto: un'indagine sul ruolo dei bias cognitivi nei processi decisionali dei progettisti
}

\author{
VALERIA BELVEDERE
}

\begin{abstract}
Obiettivo del paper: L'articolo affronta il tema dell'overdesign, ovvero della tendenza dei progettisti a sviluppare prodotti/servizi superiori rispetto alle effettive esigenze dei clienti. In particolare, l'obiettivo è verificare se $e$ in che misura i bias cognitivi determinino questo fenomeno e quale ruolo svolgano $i$ sistemi di pricing del prodotto e di misura delle prestazioni dei Progettisti rispetto ai meccanismi distorsivi delle decisioni.

Metodologia: $\grave{E}$ stata condotta una survey che ha consentito di raccogliere 149 questionari utili, fra i progettisti di aziende operanti in settori design-based.

Risultati: I dati raccolti, sottoposti ad un'analisi fattoriale, hanno evidenziato l'esistenza di 3 forme di overdesign e di 6 categorie di bias cognitivi. Attraverso un'analisi di regressione, si è verificato che i bias dei progettisti costituiscono driver significativi delle tre forme di overdesign. L'impatto dei sistemi di pricing e di misura delle prestazioni sembra invece essere piuttosto limitato.

Limiti della ricerca: Questo lavoro presenta i limiti peculiari degli studi basati su survey. In particolare, al fine di verificare la validità delle evidenze emerse, occorrerebbe ampliare il campione oggetto di analisi e, soprattutto, replicare lo studio in contesti settoriali diversi rispetto a quelli osservati.

Implicazioni pratiche: Questo studio evidenzia il ruolo dei bias cognitivi nell'influenzare l'overdesign e sottolinea la necessità di comprendere come operare un "debias" dei processi decisionali. A questo proposito, il presente lavoro ha verificato l'efficacia solo di un ristretto numero di soluzioni che precedenti studi ipotizzano essere utili in questi contesti. Risulta, perciò, necessario approfondire lo studio degli strumenti utili a mitigare i bias cognitivi e le relative conseguenze sull'overdesign.

Originalità del paper: Questo studio ha approfondito un aspetto dei processi di innovazione, ovvero l'overdesign, utilizzando la prospettiva "comportamentale", che costituisce un filone recente negli studi sullo sviluppo del nuovo prodotto.
\end{abstract}

Parole chiave: innovazione; overdesign; bias cognitivi; behavioral operations; survey

Purpose of the paper: This paper deals with the problem of overdesign, i.e. the attitude of designers to develop products/services that exceed customers' needs. Namely, the objective of the paper is twofold: 1) to check whether and to what extent cognitive biases drive

Assistant Professor di Economia e Gestione delle Imprese - Università Bocconi

e-mail: valeria.belvedere@sdabocconi.it 
overdesign; 2) to check whether some pricing policies and designers' performance measurement systems can mitigate cognitive biases.

Methodology: A survey has been conducted among designers of design-based companies; 149 usable questionnaires have been collected.

Findings: A factor analysis has been carried out that has highlighted 3 dimensions of overdesign and 6 typologies of cognitive biases. Then, through a regression analysis it has been confirmed that cognitive biases drive the 3 dimensions of overdesign. Empirical evidence shows that the impact of pricing policies and performance measurement systems is rather weak.

Research limits: This paper suffers from the limits peculiar to survey-based studies. In particular, to confirm the evidence stemming from this paper, it could be worthwhile widening the sample and replicating this analysis in other industries.

Practical implications: This paper highlights the relevance of cognitive biases as drivers of overdesign and points out the necessity to conduct further analysis in order to understand how to "debias" the decision making process of the designers. In this regard, the effectiveness of specific pricing policies and performance measurement systems has been tested, but the outcomes only partially support this hypothesis.

Originality of the paper: This paper has analyzed a specific feature of the new product development process, i.e. overdesign, through a behavioral perspective, which is considered an innovative approach to the research in the field of innovation and operations management.

Key words: innovation; overdesign; cognitive bias; behavioral operations; survey

\section{Introduzione}

Lo sviluppo del nuovo prodotto costituisce un processo cruciale per la competitività delle imprese poiché da esso dipende la capacità di lanciare sul mercato prodotti/servizi capaci di differenziare l'offerta aziendale rispetto alla concorrenza (Grando et al., 2010). Questa considerazione ha determinato nel corso degli ultimi decenni un significativo miglioramento delle caratteristiche tecniche dei prodotti (si pensi ai settori automobilistico e dell'elettronica di consumo), un marcato ampliamento della gamma e una maggiore frequenza di aggiornamento della stessa. Se da un lato queste scelte possono creare valore per il cliente, nella misura in cui esso vede di fronte a sé una più ampia scelta (Giaccone e D'Allura, 2011), dall'altro esse determinano nuovi rischi e patologie per l'impresa, che portano con sé costi inattesi e talvolta anche un maggiore rischio di fallimento nel processo di sviluppo del nuovo prodotto. Una di queste patologie di recente identificata è l'overdesign, ovvero la tendenza dei progettisti a sviluppare prodotti/servizi con performance e/o funzionalità superiori rispetto alle effettive esigenze dei clienti (Coman e Ronen, 2009; Ronen e Pass, 2008). Tale fenomeno sembra essere piuttosto diffuso in vari settori, fra cui l'elettronica di consumo e lo sviluppo di tecnologie informatiche, ed è fonte di numerosi problemi, come il ritardo nel lancio di nuovi prodotti, costi di sviluppo superiori rispetto ai budget iniziali e, talvolta, anche una certa insoddisfazione dei clienti, che non sempre apprezzano funzionalità eccessivamente sofisticate (Torrisi, 2011). Pur a fronte di queste evidenze, la tendenza all'overdesign nel processo di Sviluppo del Nuovo Prodotto 
(SNP) non è stata ancora oggetto di sufficienti approfondimenti e, in particolare, non risultano essere stati descritti e testati in maniera esaustiva i possibili driver di questo fenomeno (Coman e Ronen, 2009).

All'interno di questo quadro di riferimento, il presente lavoro intende contribuire alla discussione sulle determinanti di questa tendenza all" "eccesso", ponendosi l'obiettivo di verificare se e in che misura l'overdesign sia determinato dai bias cognitivi peculiari degli addetti alla Progettazione. Nel corso degli ultimi anni, infatti, negli studi di operations e innovation management si è affermato un nuovo filone di ricerca di tipo "comportamentale" (Gino e Pisano, 2008), che cerca di verificare quale ruolo abbiano i meccanismi distorsivi dei processi decisionali nelle attività tipicamente svolte da chi opera nelle funzioni tecnico-produttive. Precedenti contributi, specie di matrice psicologica, hanno evidenziato il ruolo cruciale dei cosiddetti bias cognitivi, con studi che mettono in discussione i paradigmi della razionalità su cui poggiano tradizionalmente le teorie economiche e di management (Kahneman e Tversky, 1981; 1979; 1974). L'ipotesi che questo studio intende testare è che una parte significativa del fenomeno dell'overdesign dipenda da una serie di bias cognitivi riscontrabili fra i Progettisti.

Nei paragrafi successivi, si descriverà il quadro teorico di riferimento da cui discendono la domanda di ricerca e le ipotesi. Successivamente, si illustrerà lo studio empirico condotto e si delineeranno le principali conclusioni e le implicazioni manageriali.

\section{Il quadro teorico di riferimento}

\subsection{Una definizione di Overdesign}

Il fenomemo dell'overdesign è stato definito come segue: “...designing and developing products or services beyond what is required by the specifications and/or the requirements of the customer or the market" (Coman e Ronen 2009; Ronen e Pass, 2008). Muovendo da questa definizione, è possibile affermare che la tendenza a proporre un'offerta "ridondante" rispetto alle effettive esigenze del cliente porta varie conseguenze, che possono essere considerate altrettante dimensioni dell'overdesign. Da un lato, infatti, questo particolare fenomeno può sostanziarsi in un'ampiezza di gamma superiore rispetto alla reale esigenza di varietà dei potenziali clienti. Dall'altro, può determinare il lancio di prodotti le cui caratteristiche o performance sono superiori a ciò per cui il cliente target è disposto a pagare. Inoltre, le imprese esposte al problema dell'overdesign spesso soffrono anche di una strutturale difficoltà a rispettare le scadenze dei progetti di SNP. Infatti, la tendenza ad arricchire il nuovo prodotto con caratteristiche eccessive, nonché a lavorare su iniziative non sempre proficue sotto il profilo commerciale, determina un'eccessiva saturazione delle risorse impiegate e un'implicita giustificazione, da parte dei Progettisti, dei ritardi dovuti alla volontà di "migliorare" il prodotto. I rischi indotti da questi comportamenti sono particolarmente critici nei settori per $\mathrm{i}$ 
quali le performance di tempo sono un'importante leva per la competizione (Grando, 2002; Blackburn, 1991).

Per quanto concerne l'ampiezza di gamma, Ulrich (2010) sostiene che essa discenda dal vario combinarsi di tre tipologie di attributi che qualificano il prodotto, detti di fit, taste e quality. I primi sono definiti come segue "[Fit attributes]...are those for which the user's preference exhibits a single strong peak for a single value of the attribute, with satisfaction falling off substantially as the artifact diverges from this value" (Ulrich, 2010; p. 115). È il caso, per esempio, della taglia di un capo di abbigliamento, per la quale chiaramente ciascun cliente ha una preferenza esclusiva. Gli attributi di taste, invece, sono quelli per cui l'acquirente ha una scala graduata di preferenze, rispetto alle varie opzioni disponibili (per esempio, il colore di un'automobile). Infine, gli attributi di qualità riguardano performance che i clienti preferirebbero sempre ricevere al massimo dell'intensità possibile, se ciò non avesse ripercussioni sul prezzo del bene (per esempio, il consumo di carburante in un veicolo).

Se si descrive l'ampiezza di gamma in funzione del vario combinarsi dei suddetti attributi, è possibile ottenere almeno due diverse misure (Ulrich, 2010). Una riguarda le cosiddette stock keeping units (SKU), ovvero il numero complessivo dei codici prodotto che l'azienda è in grado di offrire al cliente. L'altra misura, invece, concerne il numero di caratteristiche/funzionalità (ovvero, di attributi) che qualificano il singolo prodotto. Una conseguenza di ciò è che, a parità di SKU, un'azienda può proporsi al mercato con un'offerta di fatto più ricca poiché i suoi articoli, presi singolarmente, sono caratterizzati da una dotazione superiore di attributi. Ciò, da un punto di vista produttivo, può avere ricadute significative in termini di maggiore complessità, specie se i prodotti presentano una distinta base molto profonda (Ramdas et al., 2003; Randall e Ulrich, 2001).

$\mathrm{Va}$, inoltre, considerato che l'offerta aziendale può superare le effettive esigenze del cliente non solo per la gamma o per il numero eccessivo di funzionalità peculiari dei singoli articoli, ma anche perché i prodotti possono presentare prestazioni settate su livelli eccessivamente elevati. Ciò può accadere in particolare per gli attributi di qualità (per esempio, la durata media di un utensile). A questo va aggiunto che anche il design del prodotto, ovvero le sue caratteristiche estetico/formali, possono costituire un aspetto capace di generare overdesign. Questo fenomeno è testimoniato dal coinvolgimento sempre più diffuso dei designer nel processo di SNP (Perks et al., 2005) che, tuttavia, non avendo una chiara comprensione delle implicazioni tecnico-produttive delle proprie scelte progettuali, spesso finiscono col prendere decisioni discutibili sotto il profilo economico (Di Stefano, 2006). Sebbene sia ampiamente provato che la superiorità estetica e formale del prodotto incida positivamente sulle prestazioni economiche dell'impresa (Talke et al., 2009; Hertenstein et al., 2005) e che i clienti riconoscano e apprezzino la presenza di elementi artistici e di design nel prodotto (Kristensen et al., 2012; Patrick and Hagtvedt, 2011; Gabrielsen et al., 2010; Hagtvedt e Patrick, 2008), risulta comunque difficile giungere ad una quantificazione oggettiva di questo specifico attributo 
(Noble e Kumar, 2010; Bloch, 1995). Rimane, tuttavia, indiscutibile come esso possa costituire una delle manifestazioni dell'overdesign.

\subsection{Overdesign e bias cognitivi}

Gli studi ad oggi condotti sull'overdesign ne identificano due principali cause:

- problemi organizzativi: alcuni meccanismi, fra cui i sistemi di misura delle prestazioni e le politiche di determinazione del prezzo dei prodotti, possono condurre all'overdesign (Ronen e Pass, 2008). Esiste, infatti, un'ampia letteratura che ha evidenziato come i criteri di valutazione dei singoli e dei gruppi ne possano determinare i comportamenti e le scelte (Jorgensen e Messner, 2010; Davila, 2000; Nixon, 1998); questo vale naturalmente anche per i Progettisti. Se questi ultimi sono misurati in funzione del loro contributo al lancio dei nuovi prodotti, inevitabilmente la gamma offerta tenderà a crescere al di là delle effettive esigenze del mercato. Anche il modo con cui è calcolato il prezzo di un nuovo prodotto può avere un impatto rilevante. Se, infatti, il prezzo è funzione del costo di produzione e di sviluppo, il Progettista tenderà ad arricchire il prodotto, perché questo meccanismo dà l'illusione che la marginalità del prodotto sia garantita;

- problemi comportamentali: come asserito da Herbert Simon (1957), gli uomini sono dotati di una "razionalità limitata", che non consente loro di raggiungere in tempi ragionevoli soluzioni ottime ai problemi con cui si confrontano. La tendenza a comportarsi da ottimizzatori, invece, è molto diffusa fra i Progettisti, i quali spesso sono animati dalla volontà di sviluppare il miglior prodotto possibile. Questo comportamento è giustificato dalla volontà di anticipare future evoluzioni del mercato o di raggiungere il numero più ampio possibile di clienti. In realtà, le conseguenze più immediate del lancio di un prodotto "eccessivo" sono un time to market troppo lungo, costi di sviluppo superiori al budget, talvolta anche una bassa soddisfazione del cliente rispetto alle prestazioni del prodotto e, persino, costi di gestione dei resi piuttosto elevati (Coman e Ronen, 2009). L'approccio all'ottimizzazione si reputa abbia origine in un problema culturale, poiché i Progettisti spesso hanno una modesta conoscenza del mercato e reputano che il proprio successo professionale dipenda dalle prestazioni tecniche dei loro prodotti piuttosto che dal loro successo economico.

Mentre il ruolo dei problemi organizzativi è stato oggetto di numerosi studi, minore sembra essere l'attenzione dei ricercatori verso l'impatto che i problemi comportamenti possono avere sulla tendenza all'overdesign. A questo proposito, nel corso degli ultimi anni la ricerca sui temi di gestione dell'innovazione e della produzione ha cominciato ad adottare una nuova prospettiva di analisi, mutuata dagli studi di matrice psicologica di Kahneman e Tversky (Gino e Pisano, 2008). I due autori, attraverso un'ampia serie di esperimenti, hanno dimostrato che il comportamento decisionale degli individui è influenzato da numerosi bias cognitivi ed euristiche, in particolare, l'ancoraggio, la disponibilità e la rappresentatività 
(Kahneman e Tversky, 1979; 1974), che determinano decisioni irrazionali, contraddittorie e controintuitive.

Queste evidenze sono state oggetto di approfondimento negli studi di management, al fine di verificare se anche nelle imprese i fenomeni sopra descritti determinino conseguenze significative. A questo proposito, gli studi di Lovallo e Sibony (2010) e Kahneman et al. (2011) hanno portato a un'accurata descrizione dei bias cognitivi peculiari dei contesti aziendali, e hanno sottolineato la necessità di comprendere come operare un "debias" dei processi decisionali manageriali. In particolare, Lovallo e Sibony (2010) identificano cinque categorie di distorsioni cognitive ricorrenti fra gli executive, ovvero:

- action-oriented bias, che si sostanziano in un eccesso di ottimismo rispetto agli esiti delle proprie scelte e che spesso portano a trascurare informazioni rilevanti ai fini di un corretto processo decisionale, come le possibili risposte dei competitor ai comportamenti dell'impresa;

- interest bias, che si verificano quando esiste un disallineamento fra gli obiettivi corporate e quelli funzionali;

- pattern-recognition bias, ovvero la tendenza a replicare schemi decisionali sperimentati in passato;

- stability bias, che riguardano la tendenza verso una certa inerzia in contesti di incertezza;

- social bias, ovvero la ricerca del consenso nel gruppo di appartenenza.

Kahneman et al. (2011) hanno, inoltre, illustrato come tradurre i bias descritti da Lovallo e Sibony (2010) in un self-assessment ad uso di aziende e figure manageriali interessate a verificare se e in che misura esse soffrano dei fenomeni sopra esposti. Questi due contributi appaiono di particolare rilievo rispetto all'obiettivo del presente lavoro. Si può ipotizzare, infatti, che i bias descritti da Lovallo e Sibony (2010) e da Kahneman et al. (2011) possano riscontrarsi nelle funzioni preposte allo sviluppo tecnico dei nuovi prodotti e che possano costituire un driver dell'overdesign. Infatti, i soggetti coinvolti nel processo di SNP devono necessariamente confrontarsi con una molteplicità di valutazioni e decisioni dal forte contenuto soggettivo, dato il grado elevato di incertezza tipico dei progetti di innovazione (Moenaert et al., 2010; Droge et al., 2008). Per esempio, nei settori caratterizzati da notevole varietà e variabilità dell'offerta, la dimensione del mercato potenziale di un nuovo prodotto è spesso determinata per analogia rispetto ad un articolo lanciato in passato e ritenuto comparabile (Abernathy et al., 1999). Questo approccio si basa su un meccanismo di ancoraggio, la cui efficacia è fortemente influenzata dall'esperienza dei progettisti e dalla loro capacità di selezionare dal loro "repertorio" personale di informazioni e conoscenze quelle più coerenti rispetto al problema in analisi (Lovallo et al., 2012; Kahneman e Tversky, 1979; 1974). Pertanto, l'identificazione di un'ancora inappropriata, magari indotta dalla volontà di sostenere la validità del progetto, può portare a valutazioni (e conseguenti scelte) che non trovano poi un riscontro nell'effettivo successo del prodotto sul mercato. 
Anche le dinamiche di gruppo possono esporre i processi decisionali a potenziali distorsioni cognitive. Come sostengono Reitzig and Sorenson (2013), i progettisti tendono ad essere autoreferenziali e a sviluppare un'eccessiva fiducia rispetto alle proprie capacità e a quelle del gruppo cui appartengono. La conseguenza di questi comportamenti consiste nella difficoltà a giudicare in maniera oggettiva un progetto e ad accettare l'idea di abbandonarlo anche quando si è consapevoli delle modeste probabilità di successo commerciale (Astebro et al., 2007; Muir, 2007; Gilovich, 1990).

Poichè l'overdesign è il risultato di scelte soggettive adottate in contesti di incertezza, si può quindi sostenere che esso sia causato anche dai meccanismi distorsivi dei processi decisionali tipici dei progettisti.

\section{Metodologia}

Il presente lavoro si pone l'obiettivo di verificare se e in che misura i meccanismi distorsivi dei processi decisionali dei Progettisti possano determinare l'overdesign.

Come sottolineato nel precedente paragrafo, gli studi sul tema in oggetto portano a ritenere che specifici problemi comportamentali possano causare questo fenomeno (Coman e Ronen, 2009; Ronen e Pass, 2008). Inoltre, recenti contributi che si collocano nel filone degli studi "comportamentali" sottolineano il ruolo spesso cruciale dei bias cognitivi e delle euristiche nei processi decisionali aziendali, evidenziando come molti errori o insuccessi nelle imprese siano di fatto attribuibile a questi problemi (Kahneman et al., 2011; Lovallo e Sibony, 2010). Quindi, è stata formulata la seguente ipotesi di ricerca:

H1: l'overdesign è tanto più accentuato quanto maggiore è l'intensità dei bias cognitivi rilevati fra i Progettisti

Si veda, in proposito, la schematizzazione nella Figura 1.

Fig. 1: Schema di riferimento

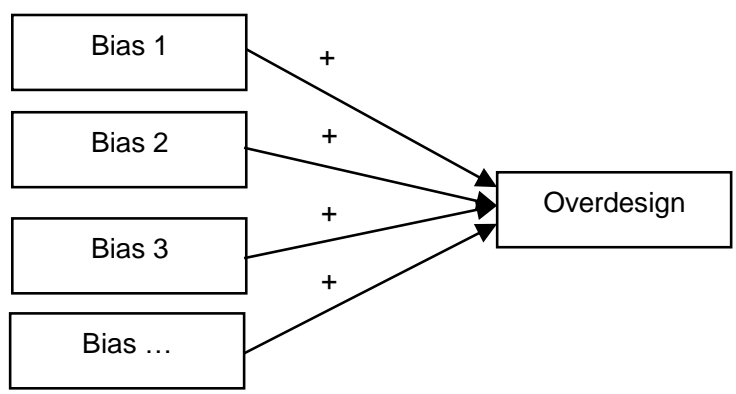

Fonte: ns. elaborazioni 
Inoltre, precedenti studi (Costabile e Cariola, 2011; Coman e Ronen, 2009; Ronen e Pass, 2008) suggeriscono che la presenza di un sistema di pricing del prodotto basato sull'analisi del valore e l'uso di opportuni indicatori di performance dei Progettisti possano attenuare la tendenza all'overdesign. Si può assumere che ciò avvenga poiché tali meccanismi aiutano a superare alcuni bias, in particolare quelli generati dal disallineamento tra gli obiettivi funzionali e quelli aziendali. Pertanto, si è formulata la seguente seconda ipotesi:

H2: l'intensità dei bias cognitivi si riduce in presenza di un sistema di pricing del prodotto basato sull'analisi del valore e di opportuni indicatori delle prestazioni dei Progettisti

Per verificare queste ipotesi, è stata condotta una survey attraverso la somministrazione di un questionario (Forza, 2002), progettato al fine di misurare l'incidenza dell'overdesign e dei bias cognitivi peculiari dei Progettisti, nonché la presenza in azienda delle condizioni che possono attenuare tali bias (sistemi di pricing e di misura delle prestazioni).

L'overdesign e i bias cognitivi sono stati misurati chiedendo ai compilatori di esprimere una valutazione su una scala di Likert da 1 (totale disaccordo) a 7 (totale accordo) riguardo a 25 affermazioni riportate nella Tabella 1.

In particolare, le prime due si basano su Ronen e Pass (2008) e Coman e Ronen (2009), secondo cui l'overdesign tipicamente si associa ad un allungamento dei tempi di SNP e a sistematici ritardi nel lancio del nuovo prodotto. Anche gli statement 3, 4 e 5 sono mutuati dai contributi di Ronen e Pass (2008) e Coman e Ronen (2009) e dalla vasta letteratura in tema di "product form", secondo cui i Progettisti tendono ad arricchire i propri prodotti con caratteristiche, funzionalità e contenuti estetici o perché reputano che ciò aiuti ad anticipare bisogni futuri, o semplicemente per una scarsa conoscenza del mercato (Perks et al., 2005). Gli statement 6 e 7, infine, fanno riferimento al tema dell'ampiezza di gamma e della frequenza di rinnovamento della stessa (Ulrich, 2010).

Le affermazioni dalla 8 alla 25 , invece, costituiscono una contestualizzazione rispetto al processo di SNP dei bias cognitivi descritti da Lovallo e Sibony (2010) e da Kahneman et al. (2011). Le valutazioni fornite dai rispondenti in relazione allo statement V18 sono state poi invertite, poiché in questo caso il valore 7 esprime una bassa intensità del bias in oggetto, mentre il valore 1 si associa ad alti gradi di intensità. 
Tab. 1: Statement per Overdesign e Bias Cognitivi

\begin{tabular}{|c|c|c|}
\hline \multirow[t]{7}{*}{ Overdesign } & V1 & I nostri progetti di SNP si concludono sempre in ritardo \\
\hline & V2 & Le scadenze intermedie dei nostri progetti di SNP non sono mai rispettate \\
\hline & V3 & $\begin{array}{l}\text { I nostri prodotti sono sempre nettamente più avanti rispetto a quelli dei principali } \\
\text { concorrenti }\end{array}$ \\
\hline & V4 & $\begin{array}{l}\text { Nel progettare un nuovo prodotto, si cerca sempre di soddisfare le esigenze non solo } \\
\text { di uno specifico target di clienti, ma anche di tutti i clienti potenzialmente interessati al } \\
\text { prodotto }\end{array}$ \\
\hline & V5 & $\begin{array}{l}\text { Le caratteristiche tecniche dei nostri prodotti sono sempre superiori rispetto alle } \\
\text { effettive esigenze dei clienti }\end{array}$ \\
\hline & V6 & La nostra gamma viene sistematicamente arricchita e aggiornata con nuovi prodotti \\
\hline & V7 & II numero di prodotti che l'azienda offre al mercato è costantemente in crescita \\
\hline \multirow[t]{18}{*}{ Bias Cognitivi } & V8 & $\begin{array}{l}\text { I progettisti sono in genere molto ottimisti in relazione all'esito del progetto, in } \\
\text { particolare in relazione alla possibilità di lanciare con successo il nuovo prodotto su } \\
\text { cui stanno lavorando }\end{array}$ \\
\hline & V9 & $\begin{array}{l}\text { I progettisti sono molto sicuri delle proprie capacità. Ritengono che esse siano } \\
\text { superiori rispetto alla media del settore e che ciò sia garanzia di successo dei progetti } \\
\text { di SNP }\end{array}$ \\
\hline & V10 & $\begin{array}{l}\text { I progettisti reputano che la probabilità di successo di un nuovo prodotto non dipenda } \\
\text { dal modo con cui i concorrenti reagiranno al suo lancio }\end{array}$ \\
\hline & V11 & $\begin{array}{l}\text { Nel condurre un progetto di SNP, i progettisti tendono a perseguire obiettivi specifici } \\
\text { dell'Unità cui appartengono, invece che obiettivi rilevanti per l'azienda nel suo } \\
\text { complesso }\end{array}$ \\
\hline & V12 & Nell'attività di SNP, i progettisti manifestano un legame emotivo con il nuovo prodotto \\
\hline & V13 & $\begin{array}{l}\text { Nel condurre un progetto di SNP, i progettisti non hanno una chiara visione degli } \\
\text { obiettivi aziendali, del loro ordine di priorità e dei possibili trade-off }\end{array}$ \\
\hline & V14 & $\begin{array}{l}\text { Nel valutare l'opportunità di avviare un nuovo progetto o di lanciare un nuovo } \\
\text { prodotto, i progettisti manifestano elevato interesse verso informazioni favorevoli al } \\
\text { lancio, mentre tendono a trascurare quelle sfavorevoli }\end{array}$ \\
\hline & V15 & $\begin{array}{l}\text { Nel valutare l'opportunità di avviare un nuovo progetto o di lanciare un nuovo } \\
\text { prodotto, i progettisti cercano di confrontare la nuova situazione con casi emblematici } \\
\text { che si sono verificati nel passato, anche se non sono del tutto confrontabili }\end{array}$ \\
\hline & V16 & $\begin{array}{l}\text { Nel valutare l'opportunità di avviare un nuovo progetto o di lanciare un nuovo } \\
\text { prodotto, i progettisti richiamano spesso alla memoria storie o situazioni molto note in } \\
\text { azienda }\end{array}$ \\
\hline & V17 & $\begin{array}{l}\text { Nel valutare l'opportunità di avviare un nuovo progetto o di lanciare un nuovo } \\
\text { prodotto, i progettisti danno più importanza alla persona proponente che all'effettiva } \\
\text { qualità dell'idea }\end{array}$ \\
\hline & V18 & $\begin{array}{l}\text { Nel valutare l'opportunità di avviare un nuovo progetto o di lanciare un nuovo } \\
\text { prodotto, in azienda si acquisiscono tutte le informazioni ritenute necessarie o utili per } \\
\text { decidere correttamente }\end{array}$ \\
\hline & V19 & $\begin{array}{l}\text { Nel valutare le probabilità di successo di un nuovo prodotto (per esempio, in termini di } \\
\text { quota di mercato, time to market etc.) in azienda si parte da un valore di riferimento, } \\
\text { che poi si aggiusta con approssimazioni successive }\end{array}$ \\
\hline & V20 & $\begin{array}{l}\text { Nella gestione dei progetti di SNP, il team si dimostra molto cauto nel prendere } \\
\text { decisioni (per esempio, aggiungere nuove funzionalità al prodotto) che potrebbero } \\
\text { anche solo parzialmente ridurre i ricavi e/o i margini del nuovo prodotto }\end{array}$ \\
\hline & V21 & $\begin{array}{l}\text { Nella gestione dei progetti di SNP, il team prende spesso decisioni che determinano } \\
\text { un incrementato dei costi di SNP, se esse sono ritenute funzionali allo sviluppo di un } \\
\text { prodotto più performante }\end{array}$ \\
\hline & V22 & $\begin{array}{l}\text { Nel decidere se proseguire o meno con un progetto di SNP, si tiene conto dei costi } \\
\text { già sostenuti }\end{array}$ \\
\hline & V23 & $\begin{array}{l}\text { I team di SNP, nel prendere decisioni, seguono schemi e meccanismi decisionali già } \\
\text { sperimentati e testati in passato }\end{array}$ \\
\hline & V24 & $\begin{array}{l}\text { Nella gestione dei team di SNP, si dà molta importanza al raggiungimento del } \\
\text { consenso e alla condivisione dei piani e degli obiettivi }\end{array}$ \\
\hline & V25 & $\begin{array}{l}\text { I membri del team sono sempre d'accordo con le idee e le opinioni del loro team } \\
\text { leader }\end{array}$ \\
\hline
\end{tabular}

Fonte: ns. elaborazioni

Per quanto concerne i sistemi di misura delle prestazioni, è stato chiesto se in azienda i Progettisti sono valutati rispetto ai criteri riportati nella Tabella 2. 
Tab. 2: Criteri di valutazione della prestazione dei Progettisti

\begin{tabular}{|l|l|}
\hline V26 & Rispetto dei tempi di completamento del progetto \\
\hline V27 & Rispetto del budget assegnato \\
\hline V28 & Margine unitario generato dal nuovo prodotto \\
\hline V29 & $\begin{array}{l}\text { Numero complessivo di prodotti nuovi lanciati in un dato intervallo di tempo (per } \\
\text { esempio, un anno) }\end{array}$ \\
\hline V30 & Costo complessivo sostenuto per il completamento del progetto \\
\hline
\end{tabular}

Fonte: ns. elaborazioni

Da ultimo, è stato chiesto di specificare come sia definito il costo di un nuovo prodotto in azienda, distinguendo tra le due modalità riportate in Tabella 3.

Tab. 3: Pricing del prodotto

\begin{tabular}{|l|l|}
\hline V31 & Il prezzo è una funzione del costo di produzione del nuovo prodotto \\
\hline V32 & $\begin{array}{l}\text { Il prezzo si definisce attraverso un'analisi del valore che il cliente attribuisce al nuovo } \\
\text { prodotto }\end{array}$ \\
\hline
\end{tabular}

Fonte: ns. elaborazioni

Il questionario è stato inviato per posta elettronica all'attenzione del Responsabile della Progettazione/Ufficio Tecnico dei soci di AIPI (Associazione Italiana Progettisti Industriali) e ADI (Associazione per il design Industriale) e, successivamente, è stato effettuato un recall telefonico ad un campione di 400 imprese operanti in settori design-based, in particolare l'arredo, l'illuminotecnica e la produzione di accessori per la casa. Questa scelta è stata indotta dalla volontà di osservare il fenomeno oggetto di indagine presso realtà in cui l'innovazione di prodotto, intesa sia nella dimensione tecnico-funzionale che estetico-formale, costituisce un elemento essenziale per la competitività aziendale (Ravasi e Lojacono, 2005). I questionari raccolti e compilati in modo completo sono 149.

\section{Evidenze empiriche}

Per verificare la prima ipotesi di ricerca (H1), i dati raccolti sono stati sottoposti ad un'analisi fattoriale; successivamente è stata condotta una regressione. In aggiunta, è stata valutata l'affidabilità interna delle scale utilizzate per misurare ciascuno dei fattori emersi attraverso l'analisi fattoriale. Per ciascuno di essi è stata calcolata l'alfa di Cronbach, che ha fornito valori compresi tra 0,754 e 0,810 (in Tabella 4.b e 5.b), ottenendo dunque evidenza della validità delle scale (Nunnally, 1978). Per quanto concerne l'analisi fattoriale, sono stati considerati rilevanti esclusivamente i fattori con un Eigenvalue superiore a 1 (Hair et al., 2006), mentre i singoli statement sono stati utilizzati per l'interpretazione dei fattori solo se caratterizzati da un factor loading superiore a 0,4 rispetto ad un solo fattore (Hu e Bentler, 1999; Stevens 1986).

I risultati dell'analisi fattoriale sugli statement relativi all'overdesign sono riportati nelle Tabelle 4.a e 4.b. 
Tab. 4.a: Analisi Fattoriale su Overdesign

\begin{tabular}{|l|c|c|c|}
\hline Fattori & Eigenvalue & \% Varianza & $\begin{array}{c}\text { \% Varianza } \\
\text { cumulata }\end{array}$ \\
\hline 1 & 2,188 & 31,256 & 31,256 \\
\hline 2 & 1,460 & 20,862 & 52,118 \\
\hline 3 & 1,014 & 14,480 & 66,598 \\
\hline
\end{tabular}

Fonte: ns. elaborazioni

Tab. 4.b: Analisi Fattoriale su Overdesign

\begin{tabular}{|c|c|c|c|}
\hline & \multicolumn{3}{|c|}{ Fattori } \\
\hline & Tempi (1) & Gamma (2) & Superiorità (3) \\
\hline Alfa di Cronbach & 0,782 & 0,754 & 0,802 \\
\hline $\begin{array}{l}\text { Le scadenze intermedie dei nostri progetti di SNP non sono mai } \\
\text { rispettate }\end{array}$ & 0,887 & & \\
\hline I nostri progetti di SNP si concludono sempre in ritardo & 0,886 & & \\
\hline $\begin{array}{l}\text { II numero di prodotti che l'azienda offre al mercato è } \\
\text { costantemente in crescita }\end{array}$ & & 0,782 & \\
\hline $\begin{array}{l}\text { La nostra gamma viene sistematicamente arricchita e } \\
\text { aggiornata con nuovi prodotti }\end{array}$ & & 0,695 & \\
\hline $\begin{array}{l}\text { Nel progettare un nuovo prodotto, si cerca sempre di soddisfare } \\
\text { le esigenze non solo di uno specifico target di clienti, ma anche } \\
\text { di tutti i clienti potenzialmente interessati al prodotto }\end{array}$ & & 0,628 & \\
\hline $\begin{array}{l}\text { Le caratteristiche tecniche dei nostri prodotti sono sempre } \\
\text { superiori rispetto alle effettive esigenze dei clienti }\end{array}$ & & & 0,808 \\
\hline $\begin{array}{l}\text { I nostri prodotti sono sempre nettamente più avanti rispetto a } \\
\text { quelli dei principali concorrenti }\end{array}$ & & & 0,778 \\
\hline
\end{tabular}

Fonte: ns. elaborazioni

Come si rileva dalle Tabelle 4.a e 4.b, i fattori rilevanti sono tre e sembrano descrivere altrettante dimensioni dell'overdesign. La prima (fattore 1) concerne il tema dei ritardi nel processo SNP. La seconda (fattore 2) richiama il problema dell'ampiezza di gamma e dell'assenza di un chiaro target di mercato. La terza (fattore 3) riguarda la superiorità del prodotto rispetto alle aspettative del cliente e rispetto all'offerta dei concorrenti.

I risultati dell'analisi fattoriale relativa ai bias cognitivi sono riportati nelle Tabelle 5.a e 5.b.

Tab. 5.a: Analisi Fattoriale sui bias cognitivi

\begin{tabular}{|l|c|c|c|}
\hline Fattori & Eigenvalue & \% Varianza & \% Varianza cumulata \\
\hline 1 & 3,411 & 18,952 & 18,952 \\
\hline 2 & 1,983 & 11,018 & 29,970 \\
\hline 3 & 1,668 & 9,266 & 39,236 \\
\hline 4 & 1,371 & 7,617 & 46,853 \\
\hline 5 & 1,184 & 6,576 & 53,429 \\
\hline 6 & 1,025 & 5,693 & 59,122 \\
\hline
\end{tabular}

Fonte: ns. elaborazioni 
Tab. 5.b: Analisi Fattoriale sui Bias cognitivi

\begin{tabular}{|c|c|c|c|c|c|c|}
\hline & & & Fattor & & & \\
\hline & $\begin{array}{c}\text { Obiettivi } \\
\text { (1) }\end{array}$ & $\begin{array}{l}\text { Autoreferenzialità } \\
\text { (2) }\end{array}$ & $\begin{array}{l}\text { Overconfidence } \\
\text { (3) }\end{array}$ & $\begin{array}{l}\text { Ancoraggio e } \\
\text { rischio } \\
(4)\end{array}$ & $\begin{array}{l}\begin{array}{l}\text { Valutazione } \\
\text { economica } \\
(5)\end{array} \\
\end{array}$ & $\begin{array}{l}\text { Sunflower } \\
(6)\end{array}$ \\
\hline Alfa di Cronbach & 0,769 & 0,774 & 0,761 & 0,810 & 0,796 & - \\
\hline $\begin{array}{|lll|}\text { Nel condurre un progetto di SNP, i progettisti tendono a } \\
\text { perseguire obiettivi } & \text { specifici dell'Unità } & \text { cuil } \\
\text { appartengono, invece che obiettivi } & \text { rilevanti } & \text { per } \\
\text { l'azienda nel suo complesso } & & \\
\end{array}$ & 0,719 & & & & & \\
\hline $\begin{array}{l}\text { Nel condurre un progetto di SNP, i progettisti non } \\
\text { hanno una chiara visione degli obiettivi aziendali, del } \\
\text { loro ordine di priorità e dei possibili trade-off }\end{array}$ & 0,672 & & & & & \\
\hline $\begin{array}{l}\text { Nel valutare l'opportunità di avviare un nuovo progetto } \\
\text { o di lanciare un nuovo prodotto, i progettisti richiamano } \\
\text { spesso alla memoria storie o situazioni molto note in } \\
\text { azienda }\end{array}$ & & 0,737 & & & & \\
\hline $\begin{array}{l}\text { Nell'attività di SNP, i progettisti manifestano un legame } \\
\text { emotivo con il nuovo prodotto }\end{array}$ & & 0,671 & & & & \\
\hline $\begin{array}{l}\text { progettisti sono molto sicuri delle proprie capacità. } \\
\text { Ritengono che esse siano superiori rispetto alla media } \\
\text { del settore e che ciò sia garanzia di successo dei } \\
\text { progetti di SNP }\end{array}$ & & 0,535 & & & & \\
\hline $\begin{array}{l}\text { Nel valutare l'opportunità di avviare un nuovo progetto } \\
\text { o di lanciare un nuovo prodotto, i progettisti cercano di } \\
\text { confrontare la nuova situazione con casi emblematici } \\
\text { che si sono verificati nel passato, anche se non sono } \\
\text { del tutto confrontabili }\end{array}$ & & 0,515 & & & & \\
\hline $\begin{array}{l}\text { Nella gestione dei team di SNP, si dà molta importanzat } \\
\text { al raggiungimento del consenso e alla condivisione dei } \\
\text { piani e degli obiettivi }\end{array}$ & & & 0,710 & & & \\
\hline $\begin{array}{l}\text { progettisti sono in genere molto ottimisti in relazione } \\
\text { all'esito del progetto, in particolare in relazione alla } \\
\text { possibilità di lanciare con successo il nuovo prodotto su } \\
\text { cui stanno lavorando }\end{array}$ & & & 0,604 & & & \\
\hline $\begin{array}{l}\text { Nel valutare l'opportunità di avviare un nuovo progetto } \\
\text { o di lanciare un nuovo prodotto, i progettisti danno più } \\
\text { importanza alla persona proponente che all'effettiva } \\
\text { qualità dell'idea }\end{array}$ & & & 0,496 & & & \\
\hline $\begin{array}{l}\text { progettisti reputano che la probabilità di successo dit } \\
\text { un nuovo prodotto non dipenda dal modo con cui } \\
\text { concorrenti reagiranno al suo lancio }\end{array}$ & & & & 0,697 & & \\
\hline $\begin{array}{l}\text { team di SNP, nel prendere decisioni, seguono schemi } \\
\text { e meccanismi decisionali già sperimentati e testati in } \\
\text { passato }\end{array}$ & & & & 0,677 & & \\
\hline $\begin{array}{l}\text { Nel valutare le probabilità di successo di un nuovo } \\
\text { prodotto (per esempio, in termini di quota di mercato, } \\
\text { time to market etc.) in azienda si parte da un valore dil } \\
\text { riferimento, che poi si aggiusta con approssimazionil } \\
\text { successive }\end{array}$ & & & & 0,471 & & \\
\hline $\begin{array}{l}\text { Nella gestione dei progetti di SNP, il team si dimostrat } \\
\text { molto cauto nel prendere decisioni (per esempio, } \\
\text { aggiungere nuove funzionalità al prodotto) che } \\
\text { potrebbero anche solo parzialmente ridurre i ricavi e/o i } \\
\text { margini del nuovo prodotto }\end{array}$ & & & & & 0,678 & \\
\hline $\begin{array}{l}\text { Nel decidere se proseguire o meno con un progetto dif } \\
\text { SNP, si tiene conto dei costi già sostenuti }\end{array}$ & & & & & 0,670 & \\
\hline $\begin{array}{l}\text { membri del team sono sempre d'accordo con le idee e } \\
\text { le opinioni del loro team leader }\end{array}$ & & & & & & 0,827 \\
\hline
\end{tabular}

Fonte: ns. elaborazioni

Dall'analisi fattoriale condotta sui bias cognitivi emergono sei fattori caratterizzati da un Eigenvalue superiore a 1 (si veda la Tabella 5.a). La loro interpretazione emerge dalla lettura degli statement, così come riaggregati nella Tabella 5.b. Il primo fattore sembra raggruppare al proprio interno i bias inerenti ai problemi di disallineamento fra gli obiettivi dei Progettisti e quelli aziendali. In effetti, come già sostenuto in precedenti studi (Coman e Ronen, 2009; Ronen e Pass, 2008), i comportamenti dei Progettisti sono spesso animati da finalità di affermazione della competenze tecniche, non sempre sono in linea con gli obiettivi 
aziendali e, più nello specifico, con le scelte di posizionamento di mercato dell'impresa.

Il secondo fattore descrive, invece, un problema di autoreferenzialità dei soggetti analizzati, i quali valutano i progetti in cui sono coinvolti manifestando un legame emotivo con gli stessi e applicando criteri di scelta che replicano esperienze emblematiche vissute in passato. Questo secondo fattore sembra strettamente collegato al successivo, i cui statement evocano il problema dell'eccesso di sicurezza e della rilevanza della dimensione organizzativa. Appare, infatti, evidente che nei team preposti allo sviluppo tecnico di un nuovo prodotto molta importanza sia assegnata al conseguimento di un clima di consenso nel gruppo e che, nella valutazione dei progetti, cruciale sia la fiducia nelle capacità del team e nella credibilità del soggetto proponente. Queste evidenze sono coerenti con quanto sostenuto in letteratura circa le distorsioni cognitive indotte dall'eccesso di fiducia e dall'autoreferenzialità dei soggetti coinvolti nello sviluppo tecnico dei nuovi prodotti (Reitzig and Sorenson, 2013; Astebro et al., 2007; Muir, 2007; Gilovich, 1990).

Il quarto fattore richiama il fenomeno dell'ancoraggio, ovvero della tendenza a valutare i nuovi progetti partendo da un valore di riferimento ("ancora") sul quale effettuare dei successivi aggiustamenti (Lovallo et al., 2012; Kahneman e Tversky, 1979; 1974). La scelta dell'“ancora" e il modo con cui vengono effettuati gli aggiustamenti sono condotti attraverso il richiamo (ancora una volta) di esperienze passate, che sembrano essere evidentemente un bagaglio di conoscenze cruciale per $\mathrm{i}$ Progettisti. Le valutazioni che emergono dal processo di ancoraggio e aggiustamento possono portare a decisioni rischiose sotto il profilo economico e caratterizzate da una marcata sottovalutazione delle dinamiche di mercato.

Il fattore 5 evoca, con i suoi due statement, il problema della valutazione economica dei progetti e descrive una contraddittorietà di fondo delle scelte. Infatti, da un lato esse sembrano essere influenzate da una notevole cautela di fronte al rischio di una compromissione dei ricavi/margini associati al nuovo prodotto, dall'altro appaiono condizionate dalla volontà di non "sprecare" costi già sostenuti, sebbene ciò a volte possa determinare la prosecuzione di progetti poco profittevoli. Queste evidenze confermano la criticità di un problema discusso in letteratura, inerente alla difficoltà dei designer a valutare in modo appropriato il potenziale economico insito nei progetti, e sottolineano ancora una volta la necessità di dotare tali soggetti di un portafoglio di indicatori di performance economica del processo SNP al fine di migliorare l'efficacia del loro operato (Costabile e Cariola, 2011; Perks et al., 2005).

Il sesto fattore, infine, è descritto da un unico statement, che richiama il cosiddetto sunflower effect (Lovallo e Sibony, 2010), ovvero il ruolo determinante svolto dal leader del gruppo e la sua capacità di influenzare le idee e le opinioni dei suoi collaboratori. Questo fenomeno sembra essere strettamente connesso con il terzo fattore, che pone molta enfasi sul tema delle dinamiche di gruppo, della coesione dei membri del team e dell'importanza del proponente nella valutazione dei nuovi progetti. Ancora una volta, quindi, sembra evidenziarsi da questo studio 
un fenomeno potenzialmente distorsivo dei processi decisionali, nella misura in cui l'insieme delle dinamiche descritte può ledere l'oggettività della valutazione dei progetti di innovazione. Per testare l'ipotesi H1, è stata condotta un'analisi di regressione su ciascuna delle tre forme di overdesign sopra descritte, impiegando i 6 fattori relativi ai bias cognitivi come predittori. Gli esiti di queste analisi sono riportati nelle Tabelle 6.a, 6.b e 6.c

Tab. 6.a: Regressione su Overdesign-Tempi

\begin{tabular}{|c|c|c|c|c|c|}
\hline & \multicolumn{2}{|c|}{ Coefficienti non standardizzati } & \multirow{2}{*}{$\begin{array}{c}\begin{array}{c}\text { Coefficienti } \\
\text { standardizzati }\end{array} \\
\beta \\
\end{array}$} & \multirow[b]{2}{*}{$t$} & \multirow[b]{2}{*}{ Sig. } \\
\hline & $\beta$ & Std. Error & & & \\
\hline Costante & 0,041 & 0,081 & & 0,503 & 0,616 \\
\hline Obiettivi & 0,377 & 0,081 & 0,373 & 4,621 & $0,000^{\star \star \star}$ \\
\hline Autoreferenzialità & 0,180 & 0,083 & 0,176 & 2,177 & $0,031^{*}$ \\
\hline Overconfidence & $-0,091$ & 0,081 & $-0,091$ & $-1,124$ & 0,263 \\
\hline Ancoraggio e rischio & 0,182 & 0,082 & 0,180 & 2,229 & $0,028^{*}$ \\
\hline Valutazione economica & 0,019 & 0,081 &, 018 & 0,229 & 0,819 \\
\hline Sunflower & 0,138 & 0,082 & 0,136 & 1,678 & $0,096^{\circ}$ \\
\hline
\end{tabular}

$\mathrm{R}^{2} 0,232 ;$ Sig. $0,000^{\star \star \star}$
${ }^{\circ} \mathrm{p}$-value $<0,1 ;{ }^{*} \mathrm{p}$-value $<0,05 ;{ }^{* \star} \mathrm{p}$-value $<0,01 ;{ }^{* \star *} \mathrm{p}$-value $<0,001$

Fonte: ns. elaborazioni

Tab. 6.b: Regressione su Overdesign-Gamma

\begin{tabular}{|c|c|c|c|c|c|}
\hline & \multicolumn{2}{|c|}{ Coefficienti non standardizzati } & \multirow{2}{*}{$\begin{array}{c}\begin{array}{c}\text { Coefficienti } \\
\text { standardizzati }\end{array} \\
\beta \\
\end{array}$} & \multirow[b]{2}{*}{$t$} & \multirow[b]{2}{*}{ Sig. } \\
\hline & $\beta$ & Std. Error & & & \\
\hline Costante & 0,032 & 0,077 & & 0,420 & 0,675 \\
\hline Obiettivi & $-0,148$ & 0,078 & $-0,152$ & $-1,903$ & $0,059^{\circ}$ \\
\hline Autoreferenzialità & 0,126 & 0,079 & 0,127 & 1,598 & 0,113 \\
\hline Overconfidence & 0,412 & 0,077 & 0,426 & 5,352 & $0,000^{\star \star *}$ \\
\hline Ancoraggio e rischio & 0,149 & 0,078 & 0,153 & 1,922 & $0,057^{\circ}$ \\
\hline Valutazione economica & 0,086 & 0,077 & 0,089 & 1,116 & 0,267 \\
\hline Sunflower & $-0,024$ & 0,079 & $-0,024$ & $-0,302$ & 0,763 \\
\hline
\end{tabular}

$R^{2}$ 0,252; Sig. 0,000 ***

${ }^{\circ} p$-value $<0,1 ;{ }^{*} p$-value $<0,05 ;{ }^{* *} p$-value $<0,01 ;{ }^{* * *} p$-value $<0,001$

Fonte: ns. elaborazioni

Tab. 6.c: Regressione su Overdesign-Superiorità

\begin{tabular}{|c|c|c|c|c|c|}
\hline & \multicolumn{2}{|c|}{ Coefficienti non standardizzati } & \multirow{2}{*}{$\begin{array}{c}\begin{array}{c}\text { Coefficienti } \\
\text { standardizzati }\end{array} \\
\beta \\
\end{array}$} & \multirow[b]{2}{*}{$t$} & \multirow[b]{2}{*}{ Sig. } \\
\hline & $\beta$ & Std. Error & & & \\
\hline Costante & 0,015 & 0,080 & & 0,182 & 0,856 \\
\hline Obiettivi & 0,200 & 0,080 & 0,201 & 2,493 & $0,014^{*}$ \\
\hline Autoreferenzialità & 0,263 & 0,082 & 0,261 & 3,230 & $0,002^{* *}$ \\
\hline Overconfidence & 0,261 & 0,080 & 0,264 & 3,279 & $0,001^{\star \star}$ \\
\hline Ancoraggio e rischio & 0,055 & 0,081 & 0,055 & 0,683 & 0,496 \\
\hline Valutazione economica & $-0,161$ & 0,080 & $-0,162$ & $-2,004$ & $0,047^{*}$ \\
\hline Sunflower & 0,178 & 0,081 & 0,177 & 2,192 & $0,030^{*}$ \\
\hline
\end{tabular}

$R^{2} 0,233$; Sig. $0,000^{\star \star *}$

${ }^{\circ} p$-value $<0,1 ;{ }^{*} p$-value $<0,05 ;{ }^{* *} p$-value $<0,01 ;{ }^{* * *} p$-value $<0,001$

Fonte: ns. elaborazioni 
Come si può osservare nelle Tabelle 6.a, 6.b e 6.c, tutte e tre le manifestazioni di overdesign sono spiegate in modo significativo dai bias rilevati attraverso questo studio. Inoltre, come si può constatare osservando i coefficienti di regressione che hanno significatività statistica accettabile (p-value $<0,1)$, l'ipotesi H1 è confermata nella maggior parte dei casi ed è smentita significativamente in due. Uno concerne l'impatto del bias "Obiettivi" sulla forma di overdesign che si manifesta in un'eccessiva ampiezza di gamma (Tabella 6.b). Al riguardo, infatti, si rileva un $\beta$ negativo, da interpretare ipotizzando che la ricerca di una marcata varietà dell'offerta da parte dei Progettisti è l'effetto di una deliberata scelta aziendale in questo senso. Quindi, un elevato grado di allineamento tra obiettivi si traduce in un numero eccessivo di SKU. Il secondo caso riguarda l'impatto di "Valutazione economica" su "Superiorità" (Tabella 6.c), anch'esso caratterizzato da un coefficiente di regressione negativo. Questo si può interpretare come una tendenza alla cautela (insita nel bias), che porta a interrompere progetti di sviluppo ritenuti troppo rischiosi sotto il profilo tecnologico.

Per quanto riguarda la seconda ipotesi di ricerca $(\mathrm{H} 2)$, è stata condotta un'ANOVA volta a confrontare l'entità delle tre forme di overdesign e dei bias in aziende che hanno compiuto scelte diverse in merito ai sistemi di pricing e di misura delle prestazioni descritti nelle Tabelle 2 e 3 . La Tabella 7 riporta le sole variabili per le quali è stata rilevata una differenza statisticamente significativa, indicando il grado di significatività ed il tipo di effetto (incremento vs decremento del fenomeno) prodotto dalla presenza di specifiche misure di performance e di un sistema di pricing basato sull'analisi del valore.

Tab. 7: Effetti delle misure di performance e dei sistemi di pricing su Overdesign e Bias

\begin{tabular}{|l|l|c|c|}
\cline { 2 - 4 } \multicolumn{1}{c|}{} & Overdesign e bias & Effetto $^{(1)}$ & P-value \\
\hline Rispetto dei tempi di completamento & - Tempi & $\downarrow$ & $0,056^{\circ}$ \\
del progetto & - Gamma & $\uparrow$ & $0,069^{\circ}$ \\
& - Overconfidence & $\uparrow$ & ${ }^{\circ} 064$ \\
\hline Rispetto del budget assegnato & - Superiorità & $\downarrow$ & $0,050^{*}$ \\
\hline Margine unitario generato dal nuovo & - Superiorità & $\downarrow$ & $0,089^{\circ}$ \\
prodotto & - Rischio e Ancoraggio & $\downarrow$ & $0,075^{\circ}$ \\
\hline $\begin{array}{l}\text { Numero complessivo di prodotti } \\
\text { nuovi lanciati in un dato intervallo di } \\
\text { tempo (per esempio, un anno) }\end{array}$ & - Gamma & $\downarrow$ & $0,030^{*}$ \\
\hline $\begin{array}{l}\text { Costo complessivo sostenuto per il } \\
\text { completamento del progetto }\end{array}$ & - Valutazione economica & & \\
\hline $\begin{array}{l}\text { II prezzo si definisce attraverso } \\
\text { un'analisi del valore che il cliente } \\
\text { attribuisce al nuovo prodotto }\end{array}$ & - - & & $0,011^{*}$ \\
\hline
\end{tabular}

(1) $\uparrow$ : incremento entità del fenomeno in presenza di indicatore; $\downarrow$ : decremento entità del fenomeno

${ }^{\circ} p$-value $<0,1 ;{ }^{*} p$-value $<0,05 ;{ }^{* *} p$-value $<0,01 ; ;^{* *} p$-value $<0,001$

Fonte: ns. elaborazioni 
Come si rileva dalla lettura della Tabella 7, l'effetto dei sistemi di pricing e delle misure di prestazione sono piuttosto variegati. I primi, di fatto, risultano del tutto irrilevanti, almeno nelle imprese osservate in questa survey. I secondi, invece, incidono solo un set ristretto di forme di overdesign e di bias cognitivi, peraltro con risultati non sempre favorevoli. Appare, infatti, evidente che i primi quattro parametri di valutazione dei Progettisti producono un effetto favorevole sulla forma di overdesign più direttamente collegata all'indicatore stesso. Di conseguenza, valutare un team di SNP rispetto ai tempi di completamento del progetto riduce l'entità del fenomeno "Tempi" (ovvero la tendenza a non rispettare le scadenze). Allo stesso modo, la presenza del criterio relativo al numero di nuovi prodotti lanciati tende a ridimensionare il fenomeno "Gamma". Analoghe valutazioni riguardano il "Rispetto del Budget Assegnato" e il "Margine Unitario Generato dal Nuovo Prodotto", che riducono la tendenza alla "Superiorità", ovvero allo sviluppo di prodotti con caratteristiche eccessivamente sofisticate, il cui costo di progettazione e produzione spesso non è adeguatamente compensato dalla disponibilità alla spesa da parte dei clienti.

Ciò che si nota, tuttavia, è anche l'effetto sfavorevole del Rispetto dei Tempi di Completamento del Progetto sul fenomeno "Gamma". Si rileva inoltre che i medesimi indicatori di prestazione incidono in maniera limitata e talvolta sfavorevole sui bias. Infatti, solo "Overconfidence", "Rischio e Ancoraggio" e "Valutazione Economica" risultano influenzati da almeno un indicatore di prestazione, ma con risultati sfavorevoli sia per il primo sia per il terzo. Quindi, si può affermare che la seconda ipotesi (H2), secondo la quale l'entità dei bias è influenzata dai sistemi di pricing e di misura delle prestazioni, trova conferma solo con riferimento ad alcuni meccanismi distorsivi delle decisioni.

\section{Implicazioni manageriali e conclusioni}

Il presente lavoro riporta le evidenze emerse da uno studio volto a verificare se e in che misura l'overdesign è determinato da una serie di bias cognitivi, che possono influenzare i comportamenti e le scelte dei Progettisti, e se opportuni indicatori di performance e politiche di pricing di prodotto possono incidere sui meccanismi distorsivi delle decisioni. L'analisi condotta, sebbene relativa ad un campione che in futuro è opportuno ampliare con ulteriori rilevazioni sul campo, porta a confermare la prima delle due ipotesi. In particolare, si rileva che l'overdesign ha tre manifestazioni, tutte condizionate da varie tipologie di meccanismi distorsivi dei processi decisionali. Si conferma, quindi, l'ipotesi secondo cui l'intensità del fenomeno overdesign cresce insieme all'intensità dei bias, con due sole eccezioni riguardanti i bias relativi alle valutazioni economiche del progetto e agli obiettivi funzionali.

La seconda ipotesi di ricerca era volta a verificare se la presenza di specifici sistemi di pricing e di valutazione delle prestazioni dei Progettisti possa influenzare i suddetti bias. Questa ipotesi nasce dalla constatazione che precedenti studi sull'overdesign vedono in ciò una leva per mitigare il fenomeno in oggetto. In realtà, 
sebbene le tre forme di overdesign illustrate in questo studio sembrino essere favorevolmente influenzate dalla presenza di alcune tipologie di indicatori, meno confortanti sono i risultati inerenti alla relazione che intercorre tra pricing, indicatori di prestazione e bias cognitivi. Infatti, i sistemi di pricing sembrano essere del tutto ininfluenti, mentre modesto è l'impatto delle misure di performance, peraltro con risultati che vanno talvolta nella direzione di un'intensificazione del bias in presenza dell'indicatore.

Questi risultati portano a due considerazioni. La prima concerne il rilievo che i bias cognitivi rivestono nel processo SNP, in particolare in relazione al fenomeno dell'overdesign. In secondo luogo, occorre sottolineare come gli strumenti che precedenti studi sul tema hanno indicato per arginare la tendenza all'eccesso da parte dei Progettisti in realtà abbiano una modesta efficacia sui meccanismi distorsivi delle loro decisioni. Appare, quindi, necessario approfondire lo studio al riguardo, al fine di comprendere come operare il cosiddetto "debias" dei processi di decision making. In questo senso, un possibile approfondimento potrebbe riguardare non solo la tipologia di indicatori impiegati per valutare i Progettisti, ma anche il modo con cui il processo di valutazione si svolge. Infatti, un'ampia letteratura sul tema ha evidenziato come l'efficacia dei sistemi di misura delle prestazioni dipenda non solo dalla correttezza con cui essi sono progettati (cosa che incide sul numero e sulla natura degli indicatori rilevati), ma anche e soprattutto dal processo di misurazione (Neely, 2005; Bourne et al., 2002), ovvero da quell'insieme di attività che le organizzazioni svolgono per implementare il sistema di misure e per trarre da esso informazioni utili ad intraprendere azioni di miglioramento. Di conseguenza, appare utile verificare quale uso in concreto le aziende facciano degli indicatori formalmente rilevati e quali processi di feedback si attivino per orientare il comportamento dei progettisti. A questo proposito, occorre valutare la possibilità di condurre studi basati su casi, che per loro natura risultano più efficaci per comprendere le cause di fenomeni complessi, come quelli oggetto del presente studio, per i quali può essere proficuo il ricorso a ricerche di tipo esplorativo.

Occorre, da ultimo, sottolineare che lo studio riportato in questo lavoro presenta limiti legati alla dimensione del campione e, in particolare, ai settori in cui esso è stato condotto. I questionari, infatti, sono stati indirizzati a imprese operanti in settori design-based, dove verosimilmente una certa tendenza ad eccedere nelle caratteristiche e funzionalità dei prodotti costituisce una leva per affermarsi e anche un modo per salvaguardare l'identità del marchio.

\section{Bibliografia}

ABERNATHY F.H., DUNLOP J.T., HAMMOND J.H. (1999), A stitch in time: Lean retailing and the transformation of manufacturing-lessons from the apparel and textile industries, Oxford University Press, New York.

ASTEBRO T., JEFFREY S.A., ADOMDZA K. (2007), "Inventor Perseverance after Being Told to Quit: The Role of Cognitive Biases", Journal of behavioral decision making, vol. 20, n. 3, pp. 253-272. 
BLACKBURN J.D. (1991). Time-based competition: the next battleground in American manufacturing, Business One Irwin Homewood, Illinois.

BLOCH P.H. (1995), "Seeking the Ideal Form: Product design and Consumer Response", Journal of Marketing, vol. 59, n. 3, pp. 16-19.

BOURNE M., NEELY A., PLATTS K., MILLS J. (2002), "The success and failure of performance measurement initiatives: Perceptions of participating managers", International Journal of Operations \& Production Management, vol. 22, n. 11, pp. $1288-1310$.

COMAN A., RONEN B. (2009), "Icarus' predicament: managing the pathologies of overspecification and overdesign", International Journal of Project Management, vol. 28, n. 3, pp. 237-244.

COSTABILE M., CARIOLA A. (2011), "La misurazione delle performance dell'impresa che innova: aspetti definitori e verifiche empiriche", Sinergie, n. 64-65, pp. 89-108.

DAVILA T. (2000), "An empirical study on the drivers of management control systems' design in new product development", Accounting, Organizations and Society, vol. 25, n. 4-5, pp. 383-409.

DI STEFANO P.P. (2006), "Tolerances analysis and cost evaluation for product life cycle", International Journal of Production Research, vol. 44, n. 10, pp.1943-1961.

DROGE C., CALANTONE R., HARMANCIOGLU N. (2008), "New Product Success: Is It Really Controllable by Managers in Highly Turbulent Environments?", Journal of Product Innovation Management, vol. 25, n. 3, pp. 272-296.

FORZA C. (2002), "Survey research in Operations Management: a process based perspective", International Journal of Operations and Production Management, vol. 22, n. 2, pp. 152-194

GABRIELSEN G., KRISTENSEN T., ZAICHKOWSKY J. (2010), "Whose design is it anyway?", International Journal of Market Research, vol. 52, n. 1, pp. 89-110.

GIACCONE S.C., D'ALLURA G.M. (2011), "La risposta del mercato all'innovazione tecnologica", Sinergie, n. 67, pp. 265-286.

GILOVICH T. (1990), "Differential construal and the false consensus effect", Journal of Personality and Social Psychology, vol. 59, n. 4, pp. 623-634.

GINO F., PISANO G. (2008), "Toward a Theory of Behavioral Operations", Manufacturing \& Service Operations Management, vol. 10, n. 4, pp. 676-691.

GRANDO A. (2002), "Profili emergenti nella domanda di servizi logistici. Evidenze empiriche e traiettorie evolutive", Sinergie, n. 57, pp.3-28.

GRANDO A., VERONA G., VICARI S. (2010), Tecnologia Innovazione Operations, Egea, Milano.

HAGTVEDT H., PATRICK V. (2008), "Art Infusion: The Influence of Visual Art on the Perception and Evaluation of Consumer Products", Journal of Marketing Research, vol. 45, n. 3, pp. 379-389.

HAIR J.F., BLACK W.C., BABIN B.J., ANDERSON R.E. (2006), Multivariate Data Analysis ( $6^{\text {th }}$ ed.), Prentice Hall, Upper Saddle River, NJ.

HERTENSTEIN J.H., PLATT M.B., VERYZER R.W. (2005), "The Impact of Industrial design Effectiveness on Corporate Financial Performance", Journal of Product Innovation Management, vol. 22, n. 1, pp. 3-21.

HU L., BENTLER P.M. (1999), "Cutoff criteria for fit indexes in covariance structure analysis: Conventional criteria versus new alternatives", Structural Equation Modeling, vol. 6, n. 1, pp. 1-55. 
JORGENSEN B., MESSNER M. (2010), “Accounting and strategising: A case study from new product development", Accounting, Organizations and Society, vol. 35, n. 2, pp. 184-204.

KAHNEMAN D. (2011), Thinking, Fast and Slow, Farrar Straus Giraux, New York.

KAHNEMAN D., LOVALlO D., SIBONY O. (2011), "Before You Make That Big Decision", Harvard Business Review, vol. 89, n. 6, pp. 50-60.

KAHNEMAN D., TVERSKY A. (1974), "Judgment under uncertainty: heuristics and biases", Science, vol. 185, pp. 1124-1131.

KAHNEMAN D., TVERSKY A. (1979), "Prospect Theory: an analysis of decisions under risk", Econometrica, vol. 47, n. 2, pp. 263-291.

KAHNEMAN D., TVERSKY A. (1981), "The framing of decisions and the psychology of choice", Science, vol. 211, n. 4481, pp. 453-458.

KRISTENSEN T., GABRIELSEN G., ZAICHKOWSKY J. (2012), "How valuable is a wellcrafted design and name brand?: Recognition and willingness to pay", Journal of Consumer Behaviour, vol. 11, n. 1, pp. 44-55.

LOVALLO D., CLARKE C., CAMERER C. (2012), "Robust analogizing and the outside view: two empirical tests of case-based decision making", Strategic Management Journal, vol. 33, n. 5, pp. 496-512.

LOVALLO D., SIBONY O. (2010), "The case for behavioral strategy”, McKinsey Quarterly, vol. 2, pp. 30-43.

MOENAERT R. K., ROBBEN H., ANTIOCO M., DE SCHAMPHELAERE V., ROKS E. (2010), "Strategic Innovation Decisions: What You Foresee Is Not What You Get", Journal of Product Innovation Management, vol. 27, n. 6, pp. 840-855.

MUIR C. (2007), "Knowing When to Quit: Do Optimism and Overconfidence Cloud Inventor Judgment?", Academy Of Management Perspectives, vol. 21, n. 4, pp. 78-80.

NEELY A. (2005), "The evolution of performance measurement research: Developments in the last decade and a research agenda for the next", International Journal of Operations \& Production Management, vol. 25 n. 12, pp. 1264-1277.

NIXON, B. (1998), "Research and development performance measurement: a case study". Management Accounting Research, vol.9, n. 3, pp. 329-355.

NOBLE C.H., KUMAR M. (2010), "Exploring the Appeal of Product design: A Grounded, Value-Based Model of Key design Elements and Relationships", Journal of Product Innovation Management, vol. 27, n. 5, pp. 640-657.

NUNNALLY J.C. (1978), Psychometric theory (2 ${ }^{\text {nd }}$ ed.), McGraw-Hill, New York.

PATRICK V., HAGTVEDT H. (2011), “Aesthetic Incongruity Resolution”, Journal of Marketing Research, vol. 48, n. 2, pp. 393-402.

PERKS H., COOPER R., JONES C. (2005), "Characterizing the Role of design in New Product Development: An Empirically Derived Taxonomy", Journal of Product Innovation Management, vol. 22, n. 2, pp. 111-127.

RAMDAS K., FISHER M., ULRICH K. (2003), "Managing Variety for Assembled Products: Modeling Component Systems Sharing", Manufacturing \& Service Operations Management, vol. 5, n. 2, pp. 142.

RANDALL T., ULRICH K. (2001), "Product Variety, Supply Chain Structure, and Firm Performance: Analysis of the U.S. Bicycle Industry", Management Science, vol. 47, n. 12, pp. 1588-1604.

RAVASI D., LOJACONO G. (2005), "Managing design and designers for strategic renewal", Long range planning, vol. 38, n. 1, pp. 51-77.

REITZIG M., SORENSON O. (2013), "Biases in the selection stage of bottom-up strategy formulation”, Strategic Management Journal, vol. 34, n. 7, pp. 782-799. 
RONEN B., PASS S. (2008), Focused Operations Management: doing more with existing resources, John Wiley and Sons, New York.

SIMON H.A. (1957), Models of Man. John Wiley and Sons, New York.

STEVENS J. (1986), Applied Multivariate Statistics for the Social Sciences, Lawrence Erlbaum Associates, Hillsdale, NJ.

TALKE K., SALOMO S., WIERINGA J.E., LUTZ A. (2009), "What about design Newness? Investigating the Relevance of a Neglected Dimension of Product Innovativeness", Journal of Product Innovation Management, vol. 26, n. 6, pp. 601-615.

TORRISI S. (2011), "Innovazione tecnologica, competenze e strategie competitive delle imprese. Il caso del software", Sinergie, n. 64-65, pp. 301-329.

ULRICH K.T. (2010), Design. Creation of artifacts in society, University of Pennsylvania, Philadelphia. 